\title{
Higher order corrections to mixed QCD-EW contributions to Higgs boson production in gluon fusion
}

\author{
Marco Bonetti, ${ }^{1, *}$ Kirill Melnikov, ${ }^{1, \dagger}$ and Lorenzo Tancredi ${ }^{2, \ddagger}$ \\ ${ }^{1}$ Institute for Theoretical Particle Physics, KIT, 76128 Karlsruhe, Germany \\ ${ }^{2}$ CERN Theory Division, CH-1211 Geneva 23, Switzerland
}

(Received 3 February 2018; published 22 March 2018)

\begin{abstract}
We present an estimate of the next-to-leading-order (NLO) QCD corrections to mixed QCD-electroweak contributions to the Higgs boson production cross section in gluon fusion, combining the recently computed three-loop virtual corrections and the approximate treatment of real emission in the soft approximation. We find that the NLO QCD corrections to the mixed QCD-electroweak contributions are nearly identical to NLO QCD corrections to QCD Higgs production. Our result confirms an earlier estimate of these $\mathcal{O}\left(\alpha \alpha_{s}^{2}\right)$ effects by Anastasiou et al. [J. High Energy Phys. 04 (2009) 003] and provides further support for the factorization approximation of QCD and electroweak corrections.
\end{abstract}

DOI: 10.1103/PhysRevD.97.056017

Higgs boson production in gluon fusion is one of the central observables in Higgs physics at the LHC. This is because the majority of Higgs bosons are produced in this channel and also because the Higgs-gluon coupling is sensitive to heavy degrees of freedom that couple to gluons and receive their masses from the Higgs mechanism.

Given the importance of Higgs boson production in gluon fusion, in recent years its description by particle theorists has been provided with ever increasing accuracy. The original computations of the Higgs boson production cross section in gluon fusion at leading (LO) [1], next-toleading (NLO) [2-4], and next-to-next-to-leading order in perturbative QCD [5-7] was recently extended to one order higher [8]. The residual uncertainty of the cross section related to uncalculated higher-order QCD corrections was estimated to be of the order of two percent $[8,9]$. To fully benefit from these remarkable achievements, one needs to reconsider the many small contributions neglected in earlier calculations and study if they can change the gluon fusion cross section by a few percent.

A comprehensive analysis of the different contributions to the Higgs boson gluon fusion cross section and their uncertainties was recently presented in Ref. [9]. Among the uncertainties are the top and bottom quark mass effects on the total cross section in higher orders of perturbative QCD,

\footnotetext{
*marco.bonetti@kit.edu

†kirill.melnikov@kit.edu

"lorenzo.tancredi@cern.ch
}

Published by the American Physical Society under the terms of the Creative Commons Attribution 4.0 International license. Further distribution of this work must maintain attribution to the author(s) and the published article's title, journal citation, and DOI. Funded by SCOAP. the truncation of the expansion used to compute the nextto-next-to-next-to-leading-order $\left(\mathrm{N}^{3} \mathrm{LO}\right)$ contribution to the gluon fusion cross section, the absence of $\mathrm{N}^{3} \mathrm{LO}$ parton distribution functions, and the uncertainty in the value of NLO QCD corrections to the so-called mixed QCD-electroweak (QCD-EW) contribution to Higgs-gluon coupling.

In this paper we focus on higher-order QCD corrections to mixed QCD-electroweak contributions. These contributions appear at two loops for the first time and they are known to increase the leading-order QCD cross section by about five percent $[10,11]$. As it is often the case in Higgs physics, it is not clear how this result changes when higherorder QCD corrections to the gluon fusion cross section are accounted for. Indeed, since the NLO QCD corrections to top-mediated Higgs production in gluon fusion are close to $\mathcal{O}(100 \%)$, it is important to know if these large corrections also apply to mixed QCD-electroweak contributions since, depending on whether they do or they do not, the cross section changes by an amount that is not negligible at the level of the precision target of a few percent.

It is difficult to compute the NLO QCD corrections to the mixed QCD-electroweak contribution. Indeed, this contribution appears at two loops for the first time, so that the computation of NLO QCD corrections to it requires the calculation of three-loop Feynman diagrams to account for virtual corrections and two-loop four-point functions to evaluate the real emission corrections. Both of these tasks are quite formidable.

To overcome this difficulty, in Ref. [12] the NLO QCD corrections to mixed QCD-EW contributions were computed in an unphysical limit where the masses of electroweak gauge bosons are considered to be significantly larger than the Higgs boson mass. For such a mass hierarchy one 
can perform a systematic large mass expansion of the corresponding Feynman graphs [13] that, effectively, turns the QCD-electroweak contribution to the Higgs-gluon coupling to a pointlike interaction vertex. It is clear that the assumed mass hierarchy is questionable and that the result can only be considered as an estimate of the NLO QCD corrections to the QCD-electroweak contribution.

According to Ref. [9], at NLO QCD, the QCDelectroweak contributions increase the gluon fusion cross section by about $5 \pm 1$ percent. The uncertainty estimate shown here refers to an attempt to quantify a possible error caused by the unphysical approximation for the Higgs and vector-boson masses employed in Ref. [12].

To improve on this result, one has to compute the NLO QCD corrections to mixed QCD-electroweak contributions to the Higgs boson production cross section in gluon fusion for the correct relation between the Higgs boson and the electroweak gauge boson masses. Recently, we made the first step in this direction by calculating the relevant threeloop virtual corrections [14]. To obtain the corrections to the gluon fusion cross section, one needs to combine this result with the real emission contributions that involve twoloop four-point functions with several mass scales; computing them is quite complicated. While work on these real emission contributions is in progress, the computation of the virtual corrections reported in Ref. [14] opens up a way to provide an estimate of the NLO QCD effects on mixed QCD-electroweak contributions that is conceptually different from what has been done in Ref. [12]. As such, it will either provide additional support for the size of mixed QCD-electroweak contributions estimated in Ref. [12] or it will indicate the potential deficiencies of such an estimate. Either of these outcomes is important for understanding the current theoretical precision on the Higgs boson production cross section in gluon fusion.

Our estimate of the NLO QCD corrections to Higgs boson production in gluon fusion is based on an observation that QCD corrections to this process can be relatively well described by the soft-gluon approximation [15-17]. The soft-gluon approximation accounts for contributions of real gluon emissions by a universal formula that depends on the leading-order cross section. The only nonuniversal piece that needs to be provided are the virtual corrections computed by us recently [14].

We now explain the details of the calculation. The Higgs boson production cross section in gluon fusion can be written as

$$
\sigma=\int \mathrm{d} x_{1} \mathrm{~d} x_{2} g\left(x_{1}, \mu\right) g\left(x_{2}, \mu\right)\left(z \sigma_{0}\right) G\left(z, \mu, \alpha_{s}\right),
$$

where $z=m_{H}^{2} /\left(s x_{1} x_{2}\right), m_{H}$ is the mass of the Higgs boson, $s$ is the center-of-mass energy squared of the hadronic collision, $\alpha_{s} \equiv \alpha_{s}(\mu)$ is the strong coupling constant, and $\mu$ denotes factorization and renormalization scales that we set equal to each other. Note that the only partonic channel that contributes in the soft approximation is the $g g$ channel.

The leading-order cross section $\sigma_{0}$ reads

$$
\sigma_{0}=\frac{\alpha_{s}^{2}}{576 \pi v^{2}} F_{0}\left(m_{H}, m_{W}, m_{Z}\right)
$$

where the form factor $F_{0}$ contains $\mathrm{QCD}$ and mixed QCD-electroweak contributions at leading order. Finally, at leading order

$$
G\left(z, \mu, \alpha_{s}\right)=\delta(1-z) .
$$

To evaluate $F_{0}$, we use the following numerical values for Standard Model parameters: $m_{H}=125 \mathrm{GeV}, m_{W}=$ $80.398 \mathrm{GeV}, m_{Z}=91.88 \mathrm{GeV}, \alpha_{\mathrm{QED}}=1 / 128.0, \sin ^{2} \theta_{W}=$ $0.2233, G_{F}=1.16639 \times 10^{-5} / \mathrm{GeV}^{2}$. We also use the Higgs field vacuum expectation value defined as $v=$ $\left(G_{F} \sqrt{2}\right)^{-1 / 2}$. We employ numerical values for $\alpha_{s}$ and gluon parton distribution functions as provided by the NNPDF30 set [18]. Specifically, we use NNPDF30 and NNPDF30 $0_{\text {nlo-as-0118 }}$ for leading- and next-to-leadingorder computations, respectively.

The leading-order cross section (2) is normalized in such a way that $F_{0}=1$ if only pure QCD contributions to the form factor $F$ are taken into account. Including also the QCD-electroweak contribution, the result reads

$$
F_{0}=\left|A_{0}\right|^{2}
$$

where

$$
A_{0}=1-\frac{3 \alpha^{2} v^{2}}{32 m_{H}^{2} \sin ^{4} \theta_{W}}\left(C_{W} A_{W}+C_{Z} A_{Z}\right)
$$

with

$C_{W}=4, \quad C_{Z}=\frac{2}{\cos ^{4} \theta_{W}}\left(\frac{5}{4}-\frac{7}{3} \sin ^{2} \theta_{W}+\frac{22}{9} \sin ^{4} \theta_{W}\right)$,

and

$$
\begin{aligned}
A_{W} & =-10.71693-i 2.302953, \\
A_{Z} & =-6.880846-i 0.5784119 .
\end{aligned}
$$

These numerical values for the mixed QCD-EW amplitudes at leading order follow from analytic calculations reported in Refs. [10,19]. To obtain $A_{W, Z}$, we consistently neglect the top quark contributions in the case of $Z$-exchange amplitudes and the third-generation contribution in the case of $W$-exchange amplitudes $^{1}$; we do exactly the same when we compute NLO

\footnotetext{
${ }^{1}$ Top quark contributions to mixed QCD-electroweak corrections are known to be tiny [11].
} 
QCD corrections to QCD-electroweak contributions as described below. We note that, according to Eq. (3), we include the square of the mixed QCD-electroweak contribution to the cross section. Numerically, this makes a tiny difference and we do it for the sake of convenience.

As explained earlier, to extend this result beyond leading order, we use the soft-gluon approximation to describe the real emission corrections. The corrections to the function $G\left(z, \mu, \alpha_{s}\right)$ then follow from the soft approximation to the $g g \rightarrow H g$ matrix element squared where, independent of the hard process, the gluon emission is described by an eikonal factor. Integrating the eikonal factor over the gluon phase space and removing the collinear singularities by renormalization of the parton distribution functions, one finds $[15,16]$

$$
\begin{aligned}
& G\left(z, \mu, \alpha_{s}\right) \\
& \quad=\delta(1-z)+\frac{\alpha_{S}}{2 \pi}\left[8 C_{A} D_{1}(z)+\left(\frac{2 \pi^{2}}{3} C_{A}+V\right) \delta(1-z)\right] .
\end{aligned}
$$

Here, $D_{1}=[\ln (1-z) /(1-z)]_{+}$is a plus distribution and $V$ is the ratio of the infrared-subtracted virtual corrections to the leading-order cross section. Note that this quantity $V$ represents the only nonuniversal contribution in the soft limit, which means that it is this quantity that may, potentially, change the relative size of electroweak corrections to the Higgs production cross section at leading and next-to-leading orders in perturbative QCD. The infraredsubtracted virtual corrections are obtained from the results for NLO QCD corrections to mixed QCD-electroweak contributions reported in Ref. [14] and from the known NLO QCD corrections to the leading-order production cross section [2]. We write

$$
V=2 \operatorname{Re}\left(A_{1, \text { fin }} A_{0}^{*}\right) /\left|A_{0}\right|^{2},
$$

where

$$
A_{1, \text { fin }}=\frac{11}{2}-\frac{3 \alpha^{2} v^{2}}{32 m_{H}^{2} \sin ^{4} \theta_{W}}\left(C_{W} A_{W}^{(1)}+C_{Z} A_{Z}^{(1)}\right),
$$

and [14]

$$
\begin{aligned}
& A_{W}^{(1)}=-11.315691-i 54.029527, \\
& A_{Z}^{(1)}=-2.975666-i 41.195540 .
\end{aligned}
$$

In principle, the above results allow us to compute the Higgs boson cross section in the soft-gluon approximation. However, it is known that the soft-gluon approximation underestimates the NLO corrections. An attempt to improve on this by constructing subleading terms was undertaken in Ref. [17]. It was argued there-using analyticity considerations in Mellin space and information on universal subleading terms in the $z \rightarrow 1$ limit that arise from soft-gluon kinematics and, also, from the collinear splitting kernels-that a useful extension of the soft approximation is obtained by replacing the plusdistribution $D_{1}(z)$ that appears in Eq. (7) with

$$
D_{1}(z) \rightarrow D_{1}(z)+\delta D_{1}(z)
$$

where

$\delta D_{1}(z)=\left(2-3 z+2 z^{2}\right) \frac{\ln ((1-z) / \sqrt{z})}{1-z}-\frac{\ln (1-z)}{1-z}$.

Note that $\delta D_{1}(z)$ is an integrable function of $z$ and not a plus distribution.

It is now straightforward to use the above results to estimate the NLO QCD corrections to the mixed QCDelectroweak contribution in the soft-gluon approximation. We take $s=(13 \mathrm{TeV})^{2}$. We use NNPDF30 sets [18] to compute the gluon fusion cross section and we use LO and NLO parton distribution functions to perform computations in respective perturbative orders. We set the values of the factorization and the renormalization scales equal to each other. The central value for both scales is taken to be $\mu=m_{H} / 2$. We note, however, that our main result-the relative change in QCD cross sections due to mixed QCDEW contributions-is practically independent of the central scale. Computing the Higgs production cross section using Eq. (1), we obtain the following results:

$$
\begin{array}{rlrl}
\sigma_{\mathrm{QCD}}^{\mathrm{LO}}=20.6 \mathrm{pb}, & & \sigma_{\mathrm{QCD} / \mathrm{EW}}^{\mathrm{LO}}=21.7 \mathrm{pb}, \\
\sigma_{\mathrm{QCD}}^{\mathrm{NLO}}=37.0 \mathrm{pb}, & \sigma_{\mathrm{QCD} / \mathrm{EW}}^{\mathrm{NLO}}=39.0 \mathrm{pb} .
\end{array}
$$

It follows from these numbers that the electroweak-QCD contributions increase both the LO and NLO cross sections by 5.3-5.5 percent. This result is consistent with the estimate of the impact of mixed QCD-EW corrections obtained in Ref. [12].

As a check on the robustness of this result, we repeat the same computation with $\delta D_{1}(z)$ in Eq. (12) set to zero. Since (as we explained earlier) by introducing $\delta D_{1}(z)$ we attempt to describe radiation beyond the soft limit, by removing it from the computation we check the sensitivity of the result to the part of the computation that is difficult to control. We find $\left(\delta D_{1} \rightarrow 0\right)$

$$
\sigma_{\mathrm{QCD}}^{\mathrm{NLO}}=30.675 \mathrm{pb}, \quad \sigma_{\mathrm{QCD} / \mathrm{EW}}^{\mathrm{NLO}}=32.3 \mathrm{pb} .
$$

It follows that also in this case the mixed QCD-electroweak contribution exceeds the QCD cross section by about 5.35 percent.

Finally, we can also check what happens if we use the exact NLO results for QCD contributions, and only employ the soft approximation to describe the mixed QCD-EW contribution. The corresponding NLO QCD cross section can be obtained with MCFM [20]. For $\mu=m_{H} / 2$, the result reads ${ }^{2}$

\footnotetext{
${ }^{2}$ All partonic channels are now included.
} 
$\sigma_{\mathrm{QCD}}^{\mathrm{NLO} \text {,full }}=35.4 \mathrm{pb}$. The change in the NLO QCD cross section caused by QCD-EW contributions is obtained from Eqs. (13) and (14). We find $\delta \sigma_{\mathrm{QCD}-\mathrm{NW}}^{\mathrm{NLO}}=1.6-2 \mathrm{pb}$, depending on whether we include the improved or unimproved soft approximation. Computing the ratio $\delta \sigma_{\mathrm{QCD}-\mathrm{EW}}^{\mathrm{NLO}} / \sigma_{\mathrm{NLO}}^{\mathrm{QCD} \text {,full }}$, we obtain $(4.7-5.5) \times 10^{-2}$, consistent with other estimates described above.

The soft approximation for real gluon emission that we employ here does not correctly describe the structuredependent radiation that arises when gluons are emitted from the "interior" of the loop amplitude. However, the contribution of the true structure-dependent radiation to the cross section is suppressed by two powers of the gluon energy relative to the soft-gluon approximation [21]. For this reason, there is a good chance that the structuredependent radiation plays a relatively minor role and that the soft-gluon approximation employed by us in this paper provides a sufficiently good description of real emission.

To conclude, we employed the soft-gluon approximation and the recent computation of three-loop virtual corrections in Ref. [14] to estimate the size of the NLO QCD corrections to mixed QCD-electroweak contributions to the gluon fusion cross section. We found that mixed QCDelectroweak contributions increase both the leading- and next-to-leading-order cross sections by 5.4 percent. This result is consistent with an estimate of these corrections presented in Ref. [12]. Further improvements are only possible if the real emission contributions are computed exactly. This is a very challenging problem that, hopefully, can be solved using the many recent advances in the technology of loop computations.

\section{ACKNOWLEDGMENTS}

We would like to thank Fabrizio Caola for useful comments. The research of K. M. was supported by the German Federal Ministry for Education and Research (Bundesministerium für Bildung und Forschung) under Grant No. 05H15VKCCA. The work of M. B. was supported by a graduate fellowship from DFG Research Training Group 1694/2 "Elementary particle physics at highest energy and precision." The research of L. T. was supported by the ERC starting Grant No. 637019 "MathAm."
[1] H. M. Georgi, S. L. Glashow, M. E. Machacek, and D. V. Nanopoulos, Phys. Rev. Lett. 40, 692 (1978).

[2] S. Dawson, Nucl. Phys. B359, 283 (1991).

[3] D. Graudenz, M. Spira, and P. M. Zerwas, Phys. Rev. Lett. 70, 1372 (1993).

[4] A. Djouadi, M. Spira, and P. M. Zerwas, Phys. Lett. B 264, 440 (1991).

[5] C. Anastasiou and K. Melnikov, Nucl. Phys. B646, 220 (2002).

[6] V. Ravindran, J. Smith, and W. L. van Neerven, Nucl. Phys. B665, 325 (2003).

[7] R. V. Harlander and W. B. Kilgore, Phys. Rev. Lett. 88, 201801 (2002).

[8] C. Anastasiou, C. Duhr, F. Dulat, F. Herzog, and B. Mistlberger, Phys. Rev. Lett. 114, 212001 (2015).

[9] C. Anastasiou, C. Duhr, F. Dulat, E. Furlan, T. Gehrmann, F. Herzog, A. Lazopoulos, and B. Mistlberger, J. High Energy Phys. 05 (2016) 058.

[10] U. Aglietti, R. Bonciani, G. Degrassi, and A. Vicini, Phys. Lett. B 595, 432 (2004).

[11] S. Actis, G. Passarino, C. Sturm, and S. Uccirati, Phys. Lett. B 670, 12 (2008).

[12] C. Anastasiou, R. Boughezal, and F. Petriello, J. High Energy Phys. 04 (2009) 003.
[13] An introduction to the large-mass expansion of Feynman diagrams can be found in V. A. Smirnov, Analytic Tools for Feynman Integrals (Springer, New York, 2012).

[14] M. Bonetti, K. Melnikov, and L. Tancredi, Phys. Rev. D 97, 034004 (2018).

[15] S. Catani, D. de Florian, and M. Grazzini, J. High Energy Phys. 05 (2001) 025.

[16] D. de Florian and J. Mazzitelli, J. High Energy Phys. 12 (2012) 088.

[17] R. D. Ball, M. Bonvini, S. Forte, S. Marzani, and G. Ridolfi, Nucl. Phys. B874, 746 (2013).

[18] R. D. Ball et al. (NNPDF Collaboration), J. High Energy Phys. 04 (2015) 040.

[19] M. Bonetti, K. Melnikov, and L. Tancredi, Nucl. Phys. B916, 709 (2017).

[20] J. M. Campbell, R. K. Ellis, and C. Williams, http://mcfm .fnal.gov.

[21] F. E. Low, Phys. Rev. 110, 974 (1958); T. H. Burnett and N. M. Kroll, Phys. Rev. Lett. 20, 86 (1968); V. Del Duca, Nucl. Phys. B345, 369 (1990); D. Bonocore, E. Laenen, L. Magnea, L. Vernazza, and C. D. White, J. High Energy Phys. 12 (2016) 121; V. Del Duca, E. Laenen, L. Magnea, L. Vernazza, and C. D. White, J. High Energy Phys. 11 (2017) 057. 